\title{
Regulation of pesticide and fertilizer rates with applied by unmanned aerial vehicle
}

\author{
Leonid Marchenko ${ }^{1}$, Igor' Smirnov ${ }^{1, *}$, and Artem Spiridonov ${ }^{1}$ \\ ${ }^{1}$ Federal Scientific Agroengineering Center VIM, 109428, 1st Institutsky passage, 5, Moscow, Russia
}

\begin{abstract}
The technology of application of pesticides, fertilizers and other agrochemicals by unmanned aerial vehicles (UAVs) in the precision farming system provides for differentiated treatment of agricultural fields with variable standards of pesticides and differentiated application of fertilizers. Existing UAVs do not provide differentiated fertilization of plants with fertilizers and differentiated treatment of plants with pesticides due to imperfect flow control systems and working fluid application rates. Substantiation of methods for regulating the flow rate and norms of working fluids of pesticides and fertilizers is based on the flow continuity equations, Bernoulli and the equation that determines the rate and dose of pesticides and fertilizers depending on the operational and technological parameters of the UAV. The paper shows that to differentiate the norms for applying the working fluid of pesticides and fertilizers, it is advisable to use methods for regulating the norms by changing the area of the outlet openings of sprayers and the step of their placement, while differentiating the norms can be carried out both by a combination of these methods and by including one or several standard sizes in the work of sprayers simultaneously.
\end{abstract}

\section{Introduction}

The technology of applying pesticides, fertilizers and other agrochemicals by unmanned aerial vehicles (UAVs) in the precision farming system provides for the differentiated treatment of agricultural fields with variable pesticide rates based on the phytosanitary state of the agrocenosis, taking into account the economic threshold of harmfulness, weed infestation, pest population density, differentiated introduction of variables doses of liquid nitrogen fertilizers in the form of foliar feeding of plants and plant growth regulators based on an agrochemical cartogram of an agricultural field [1-4].

For each elementary section of the field that requires processing, a predetermined amount of pesticide or fertilizer working fluid is introduced in an uninterrupted stream with deviations not exceeding the permissible values established by agrotechnical requirements. Differentiation of the norms of working fluids of pesticides and fertilizers within the limits established by agrotechnical requirements, according to a given program in the form of an electronic task card, is one of the main conditions for the implementation of the technological process in a precision farming system using BVS, which in turn must be

\footnotetext{
*Corresponding author: rashn-smirnov@yandex.ru
} 
equipped with automatic control systems (ACP) flow rate and automatic dosing systems (MAD).

To implement the technology, an unmanned aerial system BAS-137 VIM was developed on the basis of an unmanned aerial vehicle (UAV) of a coaxial helicopter type BVS-137 VIM (takeoff weight $280 \mathrm{~kg}$, payload $80 \mathrm{~kg}$ ) and an unmanned aerial vehicle (UAV) based on a gyroplane (takeoff weight $750 \mathrm{~kg}$, payload up to $400 \mathrm{~kg}$ ) for differentiated application of pesticides, fertilizers and other agrochemicals in the precision farming system $[5,6]$.

There is a variety of ways to regulate the flow rate and systems for automatic dosing of liquids $[7,8]$.

Regulation of the flow rate of the working fluid can be carried out in automatic mode by changing the working chamber of the pump, changing the number of revolutions of the pump motor shaft, bypassing a part of the main flow, throttling the flow through the regulating body.

For the programmed dosing of the working fluid of pesticides and fertilizers when they are introduced with the help of UAVs, a continuous-action SAD with an ACP of flow rate, with electric control of actuators can be used.

Value of the norm (dose) $\mathrm{N}$ can be defined as the integral of the flow rate of the working fluid) $\mathrm{Q}(\mathrm{t})$ for a certain period of time $(\mathrm{t} 1, \mathrm{t} 2)$ dose formation $[7,8]$ :

$$
\begin{gathered}
N=\int_{t_{1}}^{t_{2}} Q(t) d t ; \\
N=\int_{t_{1}}^{t_{2}} s(t) \omega(t) C_{i}(t) d t,
\end{gathered}
$$

where $\mathrm{s}(\mathrm{t})$ - flow section;

$\omega(\mathrm{t})$ - flow rate;

$\mathrm{Ci}(\mathrm{t})$ - concentration of the $\mathrm{i}$-th component in the flow of matter.

The proposed definition of the dose characterizes the effect on its value of the main parameters - the area and flow rate, the concentration of the substance in the flow, but does not take into account the influence of the technical and operational parameters of the technological process of introducing the working fluid.

The flow rate of the working fluid for differentiated application on the cultivated agricultural field is set based on the calculated doses of the active substance of pesticides and fertilizers for each selected elementary area of the field, which are rectangles with a width equal to the working width of application and a length equal to the length of the selected contour.

The analysis of the developed UAVs for plant protection showed that the pesticide spraying systems, as a rule, are integrated with the UAV flight control system and operate according to the specified GPS coordinates in accordance with the program for the processing of agricultural fields, however, the regulation of the working fluid flow rate is carried out by changing the operating pressure in the system or in the "on / off" mode [912]. This approach to regulating the flow rate of the working fluid is unproductive when differentiating the rates of application of working fluids of pesticides and fertilizers.

Research Objective: substantiate the ways of regulating the norms of working fluids of pesticides and fertilizers when they are applied by an unmanned aircraft.

\section{Material and methods}

The substantiation of methods for regulating the norms of working fluids of pesticides and fertilizers was based on the law of conservation of mass, energy and the equation that 
determines the rate (dose) of the working fluid of pesticides, fertilizers and agrochemicals, depending on the operational and technological parameters of the BVS.

The law of conservation of mass in the form of an equation of continuity (continuity) of the flow is represented by the equation:

$$
G=\rho \omega f=\text { const }
$$

where $\mathrm{G}$ - mass flow rate;

$\rho$ - flow medium density;

$\omega$ - average flow rate;

$f$ - cross-sectional area of flow.

The energy conservation law is written in the form of the Bernoulli equation, which expresses the energy balance of the flow:

$$
Z+\frac{P}{p g}+\frac{\rho \omega^{2}}{2 g}=\text { const }
$$

where $\mathrm{Z}$ - geometric height of the center of gravity of a given flow section, specific potential energy of a position in a given section;

$\frac{P}{p g}-$ specific potential energy of fluid pressure;

$\frac{\rho \omega^{2}}{2 g}-$ specific kinetic energy of the flow in a given section;

$\mathrm{P}$ - flow medium density;

$\mathrm{g}$ - acceleration of gravity.

The equation that determines the rate of application of the working fluid of the pesticide or fertilizer, depending on the design and technological parameters of the BVS spraying system, has the form:

$$
N=\frac{Q \times n}{B \times V}
$$

where $\mathrm{N}$ - rate (dose) of the working fluid;

$\mathrm{Q}$ - volumetric flow rate of working fluid through one atomizer;

B - working width of capture;

$\mathrm{V}$ - operating speed;

$\mathrm{n}$ - number of nozzles on the boom of the sprayer module.

For the volumetric flow rate of the working fluid Q, the flow continuity equation (3) takes the form:

$$
Q=\omega \times f_{\mathrm{cn}}
$$

where fсп - compressed flow area.

$$
f_{\text {сп }}=\varepsilon \times f
$$

where $\varepsilon$ - jet compression ratio,

$f$ - outlet area.

Flow rate $\omega$ can be obtained from the Bernoulli equation:

$$
\omega=\sqrt{\frac{2 \Delta P}{(1 \oplus \xi) \rho}},
$$

where $\Delta P$ - pressure drop in front of the atomizer;

$\xi$ - total drag coefficient;

$\rho$ - flow medium density. 
Coefficient $\xi$ predetermines the speed ratio:

$$
\varphi=\frac{1}{(1+\zeta)^{0,5}}
$$

Taking into account (7), (8), (9) flow equation (6) takes the form:

$$
Q=\varphi \varepsilon f \sqrt{\frac{2 \Delta P}{\rho}}
$$

Product of odds $\varepsilon$ and $\omega$ gives the nozzle flow coefficient $\mu$. Coefficients $\mu, \varphi$ and $\varepsilon$ depend on the Reynolds, Froude and Weber numbers.

In practical cases, when liquid flows through holes, the influence of the Froude and Weber numbers is neglected and the coefficients are calculated $\varepsilon, \varphi$ and $\mu$ function of number Re. In the range of numbers $\mathrm{Re}=104 \div 106$ the flow coefficient, therefore, and the velocity coefficient in round holes, vary within insignificant limits, they can be considered constant values [13].

For slotted nozzles, the flow rate depends on the ratio of the length of the nozzle to its width $[14,15]$.

Substituting into equation (4) the value of the flow rate from equation (9), we obtain an expression characterizing the rate of application of the working fluid, depending on the flow characteristics of the nozzles, design and flight technical parameters of the UAV.

$$
N=n \mu f \frac{\sqrt{\frac{2 \Delta P}{\rho}}}{(B \times V)}
$$

Given that the attitude $\mathrm{B} / \mathrm{n}$ determines the step 1 the arrangement of sprayers on the boom of the UAV sprayer module, expression (10) takes the form:

$$
\mathrm{H}=\mu f \frac{\sqrt{\frac{2 \Delta P}{\rho}}}{(l \times V)}
$$

\section{Results and discussion}

Expressions (10) and (11), determining the rate of application of the working fluid, show that with the differentiated application of BVS pesticides, fertilizers, plant growth regulators and other agrochemicals, the regulation of their rates (doses) can be carried out by changing the number of sprayers on the sprayer module boom, the area and, accordingly, the nominal diameter of the nozzle outlet sprayer, differential working pressure in front of the nozzles, UAV operating speed, working width, the ratio of the working width to the number of nozzles, characterizing the distance between adjacent nozzles - the spacing of the nozzles on the boom.

It should be noted that the regulation of the application rates by changing the working width of the working in a practical aspect does not make sense.

The analysis of the regulation range of norms (doses) of working fluids of pesticides and nitrogen fertilizers (UAN) was carried out in relation to BVS-137 VIM. The working width of the grip B was taken to be equal to the design length of the boom of the BVS-137 VIM sprayer module, which is $5 \mathrm{~m}$. The main parameters were changed within the following limits: operating speed $20 \leq \mathrm{V} \leq 90 \mathrm{~km} / \mathrm{h}$; spacing of nozzles on the sprayer module boom $0.1 \leq 1 \leq 0.6 \mathrm{~m}$; pressure drop in front of the nozzle $0.2 \leq \Delta \mathrm{P} \leq 0.8 \mathrm{MPa}$; nozzle outlet area $0,452 \times 10-7 \leq \mathrm{f} \leq 5,027 \times 10-7 \mathrm{~m} 2$ with the nominal diameter of the outlet 0,2710-3 $\leq \mathrm{d} \leq 0,8 \times 10-3 \mathrm{~m}$ when applying pesticide working fluid; $4,0 \times 10-7 \leq \mathrm{f} \leq$ 
$14,61 \times 10-7 \mathrm{~m} 2$, when applying a working solution of liquid nitrogen fertilizer with the nominal diameter of the outlet $0,72 \times 10-3 \leq \mathrm{d} \leq 1,36 \times 10-3 \mathrm{~m}$. When one of the parameters changed, the values of the other three were fixed: $\mathrm{V}=40 \mathrm{~km} / \mathrm{h}, \Delta \mathrm{P}=0,4 \mathrm{MPa}, 1=0,25 \mathrm{~m}, \mathrm{f}$ $=2,165 \times 10-7 \mathrm{~m} 2$. Slot sprayer flow rate $\mu=0,85$. Density of pesticide working fluid $\rho=$ $1000 \mathrm{~kg} / \mathrm{m} 3$, average density of the working solution of nitrogen fertilizer $\rho=1200 \mathrm{~kg} / \mathrm{m} 3$.

Using expressions (10) and (11), one-factor dependences were constructed $\mathrm{N}=\mathrm{h}(\mathrm{V}), \mathrm{N}$ $=\mathrm{h}(\mathrm{l}), \mathrm{N}=\mathrm{h}(\Delta \mathrm{P}), \mathrm{N}=\mathrm{h}(\mathrm{f})$, presented in the figures $1,2,3,4$.

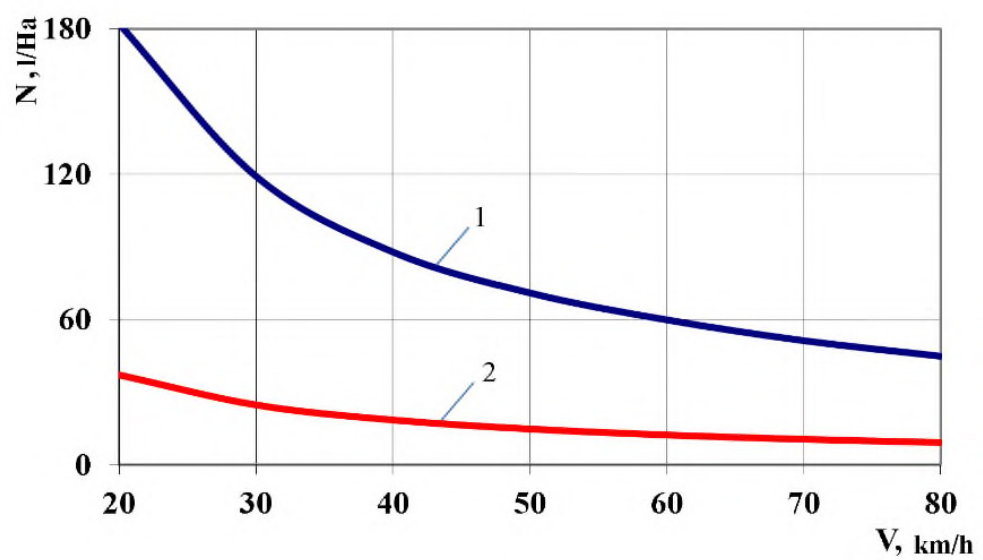

Fig. 1. Dependence of the rate of application of the working fluid on the operating speed of flight: 1 at application of pesticides; 2 - at application of nitrogen fertilizers.

Dependency Analysis $\mathrm{H}=\mathrm{h}(\mathrm{V})$ shows that in the operating range of BVS-137 VIM flight speeds from 30 to $60 \mathrm{~km} / \mathrm{h}$, the rate of the working solution of nitrogen fertilizer changes from 120 to $601 /$ ha, that is, it is halved. The rate of pesticide working fluid within $30-60 \mathrm{~km} / \mathrm{h}$ varies from about 25 to $121 /$ ha and also halves. The UAV autopilot, in accordance with a given flight program along a given trajectory, can change the operating speed and, as a consequence, the application rate is adequate for each elementary section of the cultivated field. However, the range of regulation of the rates of the working fluid consumption within the operating flight speeds does not provide the prescribed doses of nitrogen fertilization for foliar feeding of plants.

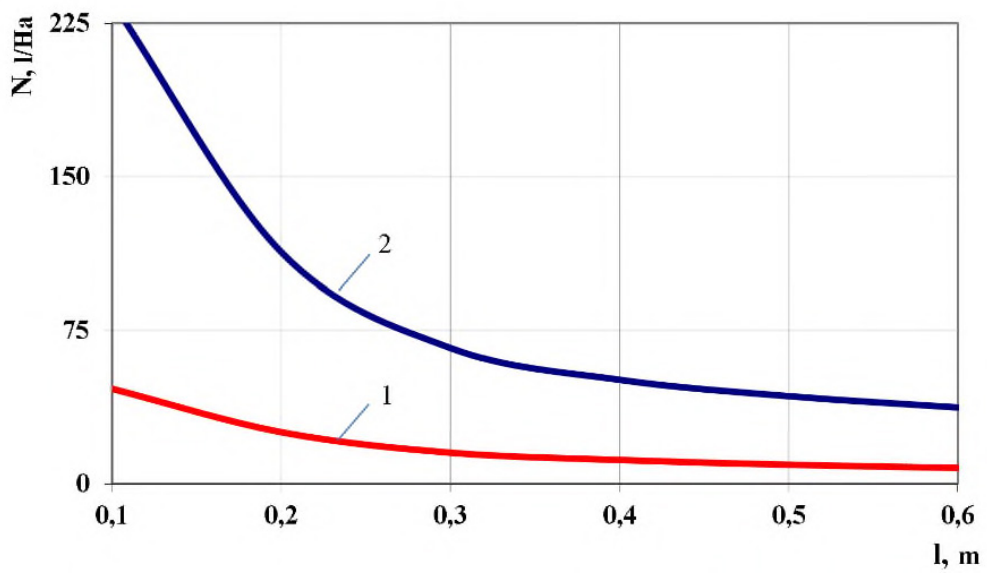

Fig. 2. Dependence of the rate of application of the working fluid on the spacing of the nozzles: 1 - at application of pesticides; 2 - at application of nitrogen fertilizers. 
Dependence $\mathrm{H}=\mathrm{h}$ (l) shows that when changing the spacing of the nozzles on the sprayer module boom from 0.6 to $0.1 \mathrm{~m}$, the application rate of the nitrogen fertilizer solution and the pesticide working fluid increases approximately six times from 37.5 to 225 1 / ha and from 7.7 to 46.41 / ha, respectively. Typically, the spacing spacing for pesticides and fertilizers is kept constant. It is possible to change the distance between adjacent nozzles by equipping them with electromagnetic shut-off valves.

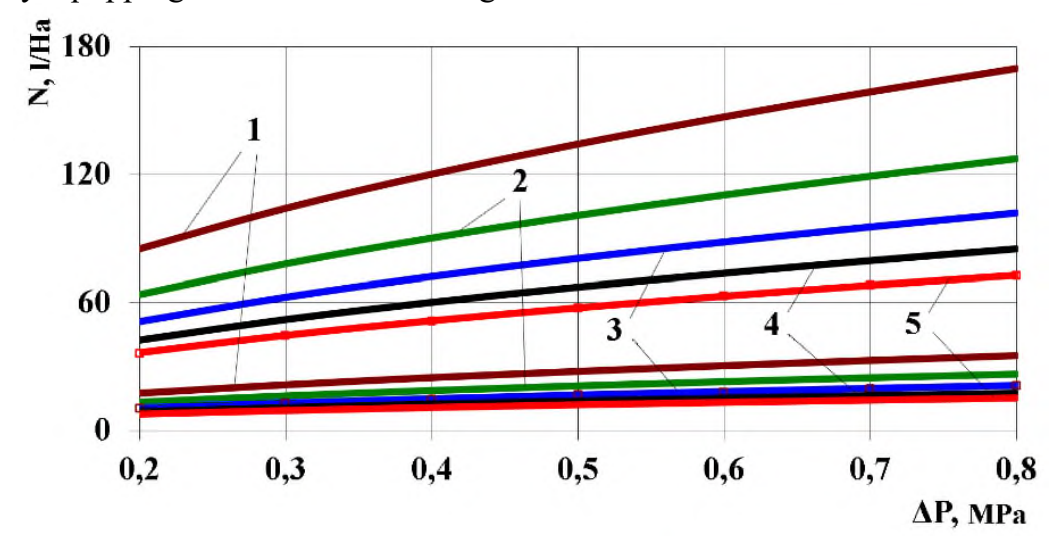

Fig. 3. Dependence of the rate of application of the working fluid of the pesticide and nitrogen fertilizer on changes in the pressure drop before the sprayer: 1 - at an operating speed of $30 \mathrm{~km} / \mathrm{h} ; 2$ at an operating speed of $40 \mathrm{~km} / \mathrm{h} ; 3$ - at an operating speed of $50 \mathrm{~km} / \mathrm{h} ; 4$ - at an operating speed of $60 \mathrm{~km} / \mathrm{h} ; 5$ - at an operating speed of $70 \mathrm{~km} / \mathrm{h}$.

The influence of the pressure drop in front of the sprayer within $0.2 \ldots 0.8 \mathrm{MPa}$ on the application rate, represented by a number of dependencies $\mathrm{N}=\mathrm{h}(\Delta \mathrm{P})$, shows that regardless of the operating flight speed, the rate of application of both the working fluid and the nitrogen fertilizer solution increases approximately twofold from 63 to 1271 / ha and from 13 to $261 /$ ha, respectively. The operating pressure drop in front of the atomizer is usually $0.3 \ldots 0.5 \mathrm{MPa}$. With an increase in the flow rate of the working fluid, for example, three times, the pressure drop must be increased nine times; therefore, it is irrational to control the flow rate of the working fluid and, accordingly, the application rate by changing the working pressure.

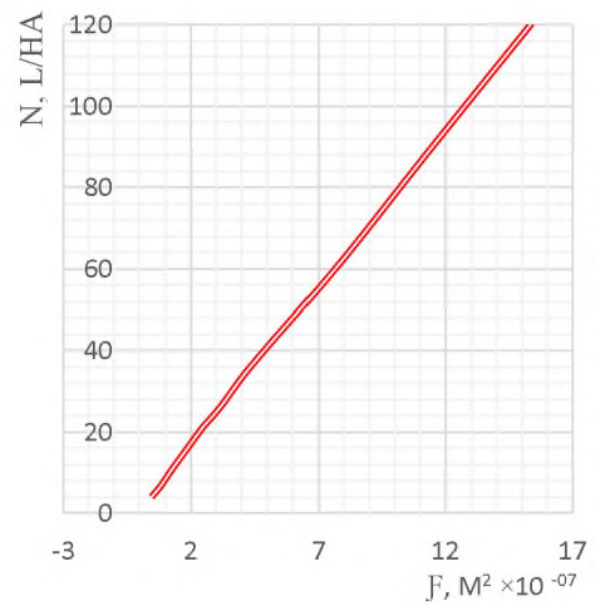

Fig. 4. Dependence of the application rate of the nitrogen fertilizer solution on the area of the spray nozzle outlet. 
Dependence $\mathrm{N}=\mathrm{h}(\mathrm{f})$, characterizing the change in the rate of application of the working fluid from the area of the outlet of the sprayer shows: with an increase in the area of the outlet of the sprayer with $0,452 \times 10-7 \mathrm{~m} 2$ (nominal diameter $0,24 \times 10-3 \mathrm{~m}$ ) to $25,45 \times 10-7$ $\mathrm{m} 2$ (nominal diameter $1,8 \times 10-3 \mathrm{~m}$ ) the application rate increases 51.5 times from 3.88 to $2001 /$ ha. This method of changing the application rate provides a fairly wide range of regulation of the working fluid rates of both pesticides and nitrogen fertilizers and is the most promising. To implement it, the boom system of the BVS sprayer module must be equipped with selective electronic units, consisting of at least four nozzles of different sizes, equipped with electro-hydraulic control systems.

Ranking the differences from maximum to minimum values of functions $\mathrm{N}=\mathrm{h}(\mathrm{V}), \mathrm{N}=$ $\mathrm{h}(\mathrm{l}), \mathrm{N}=\mathrm{h}(\Delta \mathrm{P}), \mathrm{N}=\mathrm{h}(\mathrm{f})$, presented in Figures 1,2,3,4 gives the series:

$$
N\{f\}>H\{l\}>H\{\Delta P\}=N\{V\} .
$$

From expression (12) it follows that the widest range of regulation of the rates of the working liquid of pesticides and fertilizers is provided by changing the area of the outlet of the sprayers, a slightly smaller range by adjusting the spacing of the sprayers and least of all, with practically equal ranges of rates, by changing the pressure drop in front of the sprayers and by changing the flight speed of the UAV.

\section{Conclusions}

1. Methods of regulating the flow rate and application rate of the working fluid of pesticides and fertilizers - by changing the flight speed of the BVS, the working pressure in the system of the sprayer module, the spacing of the sprayers, and the area of their outlet openings.

2. It is shown that to differentiate the rates of application of the working fluid of pesticides and fertilizers, it is advisable to use the methods of regulating the rates by changing the area of the outlet openings of the nozzles and the step of their arrangement, while the differentiation of the rates can be carried out both by a combination of these methods and by including one or several standard sizes in the operation of the sprayers at the same time. 3. It has been established that the widest range of application rate regulation from 3.88 to $2001 /$ ha is provided by changing the area of the spray nozzles and the step of their arrangement on the boom of the BVS sprayer module.

\section{References}

1. M. Ayre, V. Mc Collum, W. Waters, P. Samson, A. Curro, R. Nettle, J-A. Paschen, Ba. King, N. Reichelt, Supporting and practising digital innovation with advisers in smart farming. NJAS, Wageningen Journal of Life Sciences 90-91, 100302 (2019) ISSN 1573-5214, doi:10.1016/j.njas.2019.05.001

2. P. Radoglou-Grammatikis, P. Sarigiannidis, T. Lagkas, I. Moscholios, A compilation of UAV applications for precision agriculture, Computer Networks 172, 107148 (2020) ISSN 1389-1286, doi:10.1016/j.comnet.2020.107148

3. B.S. Faiçal, F.G. Costa, G. Pessin, J. Ueyama, H. Freitas, A. Colombo, P.H. Fini, L. Villas, F.S. Osório, P.A. Vargas, T. Braun, The use of unmanned aerial vehicles and wireless sensor networks for spraying pesticides, Journal of Systems Architecture 60, 4, 393-404 (2014) ISSN 1383-7621, doi:10.1016/j.sysarc.2014.01.004

4. H.Zhu, Y. Lan, W. Wu, W. C. Hoffmann, Y. Huang, X. Xue, J. Liang, B. Fritz, Development of a PWM Precision Spraying Controller for Unmanned Aerial Vehicles, 
Journal of Bionic Engineering 7, 3, 276-283 (2010) ISSN 1672-6529, https://doi.org/10.1016/S1672-6529(10)60251-X

5. M. Nurfaiz Abd. Kharim, A. Wayayok, A.Rashid, M. Shariff, A.F. Abdullah, E. M. Husin, Droplet deposition density of organic liquid fertilizer at low altitude UAV aerial spraying in rice cultivation. Computers and Electronics in Agriculture 167, 105045 (2019) ISSN 0168-1699, https://doi.org/10.1016/j.compag.2019.105045

6. F. Ahmad, B. Qui, X. Dong, J. Ma, X. Huang, S. Ahmed, F. Chandio, Effect of operational parameters of UAV sprayer on spray deposition pattern in target and offtarget zones during outer field weed control application. Computers and Electronics in Agriculture 170 (2020) Doi: 10.1016/j.compag.2020.105350

7. A.L.R. Oliveira, V.A. Rodriguez, R.M. de Carvalho, M.S. Powell, L.M. Tavares. Mechanistic modeling and simulation of a batch vertical stirred mill. Minerals Engineering 156, 106487 (2020) ISSN 0892-6875, doi:10.1016/j.mineng.2020.106487.

8. S. Mypati, A. Docoslis, D.P.J. Barz, Direct writing of liquids by micro dispensing: Stability and shape of laminar jets with high Froude numbers. Chemical Engineering Journal 381, 122645 (2020) ISSN 1385-8947, doi: 10.1016/j.cej.2019.122645.

9. Wen S., Zhang Q., Deng J., Lan Y., Yin X., Shan J., Design and Experiment of a Variable Spray System for Unmanned Aerial Vehicles Based on PID and PWM Control, Applied Sciences 8(12), 2482 (2018) DOI:10.3390/app8122482

10. Liu Y., Ru Y., Duan L., Qu R., Model and design of real-time control system for aerial variable spray. PLoS ONE 15(7): e0235700 (2020) DOI: 10.1371/journal.pone.0235700.

11. D. K. Giles, R. C. Billig. Development and Performance of a UAV for Crop Spraing, Chemical Engineering Transactions 44, 307-312 (2015)

12. He X. K., Bonds J., Herbst A., Langenakens J., Regent development of unmanned aerial vehicle for plant protection in East Asia. IntAgric\&BiolEng. 10(3), 18-30 (2017)

13. A. Harnsihacacha, A. Piyapaneekoon, P. Kowitwarangkul, Physical water model and CFD studies of fluid flow in a single strand tundish, Materials Today: Proceedings 5, 3, 2, Pages 9220-9228 (2018) ISSN 2214-7853, doi: 10.1016/j.matpr.2017.10.093.

14. M. M. Tareq, R. A. Dafsari, S. Jung, J. Lee, Effect of the physical properties of liquid and ALR on the spray characteristics of a pre-filming airblast nozzle, International Journal of Multiphase Flow 126, 103240 (2020) ISSN 0301-9322, doi:10.1016/j.ijmultiphaseflow.2020.103240.

15. J. Cheng, F. Zhang, T. Liu, A discontinuous Galerkin method for the simulation of compressible gas-gas and gas-water two-medium flows, Journal of Computational Physics 403, 109059 (2020) ISSN 0021-9991, doi:10.1016/j.jcp.2019.109059. 